\title{
Primary angiitis of the central nervous system in children - case series
}

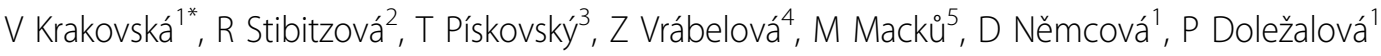 \\ From 18th Pediatric Rheumatology European Society (PReS) Congress \\ Bruges, Belgium. 14-18 September 2011
}

\section{Background}

Primary angiitis of the central nervous system (PACNS) is a rare inflammatory brain disease with variable clinical manifestations. Only limited data exist with regard to efficient diagnostic and therapeutic algorithms and disease outcomes in children.

\section{Aim}

To characterize clinical features, neuroimaging findings, treatment and disease outcome in a small cohort of Czech children with PACNS.

\section{Methods}

A retrospective chart review of patients referred for suspected PACNS was performed. MRI lesions detected at disease onset were classified as unilateral (UL), bilateral (BL), unifocal (F), multifocal (MF), angiography (MRA and/or conventional) positive (medium/large vessel disease) or negative (small vessel disease). According to the evolution of clinical manifestations and neuroimaging PACNS was classified as progressive or non-progressive. Data on treatment and follow-up were collected.

Table

\begin{tabular}{|c|c|c|c|c|c|c|c|}
\hline Patient & 1 & 2 & 3 & 4 & 5 & 6 & 7 \\
\hline $\begin{array}{l}\text { Age at onset } \\
\text { (years) }\end{array}$ & 13 & 1,5 & 12 & 16 & 5,5 & 6 & 15 \\
\hline $\begin{array}{l}\text { Time to diagnosis } \\
\text { (months) }\end{array}$ & 30 & 5 & 1 & 1,5 & 12 & 1 & 1,5 \\
\hline F/U (years) & 4 & 13 & 5 & 0,5 & 2 & 1,5 & 1 \\
\hline $\begin{array}{l}\text { Manifestation at } \\
\text { onset }\end{array}$ & generalised seizures & focal seizure, stroke & $\begin{array}{l}\text { seizure } \\
\text { sensory deficit }\end{array}$ & $\begin{array}{l}\text { sensory } \\
\text { deficit, stroke }\end{array}$ & stroke & $\begin{array}{l}\text { stroke with } \\
\text { ataxia }\end{array}$ & $\begin{array}{l}\text { seizures } \\
\text { stroke }\end{array}$ \\
\hline MRI at onset & $U L, M F$ & $U L, M F$ & $\mathrm{BL}, \mathrm{MF}$ & $\mathrm{BL}, \mathrm{MF}$ & UL,MF & $U L, F$ & UL,MF \\
\hline $\begin{array}{l}\text { Angiography at } \\
\text { onset }\end{array}$ & $\mathrm{N} / \mathrm{D}$ & posit. & posit. & posit. & posit. & posit. & neg. \\
\hline Initial therapy & AED & AED & ACA & $A C A, C S$ & 0 & ACA & IVMP, AED \\
\hline $\begin{array}{l}\text { Manifestation at } \\
\text { F/U }\end{array}$ & $\begin{array}{l}\text { seizures cognitive } \\
\text { dysfunction }\end{array}$ & $\begin{array}{l}\text { hemiparesis } \\
\text { developmental delay }\end{array}$ & normal & normal & $\begin{array}{l}\text { cognitive dysfunction, } \\
\text { hemiparesis }\end{array}$ & normal & $\begin{array}{l}\text { organic } \\
\text { psychosis }\end{array}$ \\
\hline $\mathrm{MRI}$ at $\mathrm{F} / \mathrm{U}$ & $B L, M F$ & $\mathrm{~N} / \mathrm{D}$ & $\mathrm{N} / \mathrm{D}$ & $\mathrm{N} / \mathrm{D}$ & $B L, M F$ & regression & $B L, M F$ \\
\hline Angiography F/U & neg. & N/D & $\mathrm{N} / \mathrm{D}$ & $\mathrm{N} / \mathrm{D}$ & regression & regression & neg. \\
\hline Therapy at F/U & AED, CS & $A E D, A C A$ & $A C A$ & CS & $A C A$ & $A C A$ & CYC, CS \\
\hline
\end{tabular}

$\underline{\mathrm{ACA}}$ - acetylsalicylic acid, $\underline{\mathrm{AED}}$ - anti-epileptic drugs, $\underline{\mathrm{CS}}$ - oral corticosteroid (prednisone or methylprednisolone), $\underline{\mathrm{CYC}}$ - cyclophosphamide, IVMP- intravenous methylprednisolone, N/D-

\footnotetext{
* Correspondence: viera.krakovska@gmail.com

${ }^{1}$ Paediatric Rheumatology Unit, General University Hospital in Prague, Czech Republic

Full list of author information is available at the end of the article
}

\section{() Biomed Central}




\section{Results}

Seven female patients (all Caucasian, mean age 14,8 years, median F/U 2 years) were identified (Table). Three had favourable outcome. Four $(1,2,5,7)$ had progressive disease resulting in significant impairment. Their mean interval from onset to diagnosis was 7 months. All 4 had multifocal lesions, 2 had negative angiography, 3 did not receive any anti-inflammatory therapy early in the disease course.

\section{Conclusion}

Unfavourable outcome of this patient cohort reflects potential severity as well as low physicians awareness of PACNS resulting in diagnostic delay and insufficient therapy. A working group of paediatric specialists is being formed in the Czech Republic in order to establish diagnostic and therapeutic algorithms in close interdisciplinary and multicenter collaboration.

\section{Author details}

${ }^{1}$ Paediatric Rheumatology Unit, General University Hospital in Prague, Czech Republic. ${ }^{2}$ Department of Paediatric Neurology, Thomayer University Hospital in Prague, Czech Republic. ${ }^{3}$ Department of Paediatrics, Teaching Hospital in Ostrava, Czech Republic. ${ }^{4}$ Department of Paediatric Neurology, University Hospital Motol, Prague, Czech Republic. ${ }^{5}$ Department of Paediatrics,

University Hospital in Brno, Czech Republic.

Published: 14 September 2011

doi:10.1186/1546-0096-9-S1-P94

Cite this article as: Krakovská et al.: Primary angiitis of the central nervous system in children - case series. Pediatric Rheumatology 20119 (Suppl 1):P94.

Submit your next manuscript to BioMed Central and take full advantage of:

- Convenient online submission

- Thorough peer review

- No space constraints or color figure charges

- Immediate publication on acceptance

- Inclusion in PubMed, CAS, Scopus and Google Scholar

- Research which is freely available for redistribution

Submit your manuscript at www.biomedcentral.com/submit 